\title{
Monomial ideals of minimal depth
}

\author{
Muhammad Ishaq
}

\begin{abstract}
Let $S$ be a polynomial algebra over a field. We study classes of monomial ideals (as for example lexsegment ideals) of $S$ having minimal depth. In particular, Stanley's conjecture holds for these ideals. Also we show that if $I$ is a monomial ideal with $\operatorname{Ass}(S / I)=\left\{P_{1}, P_{2}, \ldots, P_{s}\right\}$ and $P_{i} \not \subset \sum_{1=j \neq i}^{s} P_{j}$ for all $i \in[s]$, then Stanley's conjecture holds for $S / I$.
\end{abstract}

\section{Introduction}

Let $K$ be a field and $S=K\left[x_{1}, \ldots, x_{n}\right]$ be a polynomial ring in $n$ variables over $K$. Let $I \subset S$ be a monomial ideal and $I=\cap_{i=1}^{s} Q_{i}$ an irredundant primary decomposition of $I$, where the $Q_{i}$ are monomial ideals. Let $Q_{i}$ be $P_{i}$-primary. Then each $P_{i}$ is a monomial prime ideal and $\operatorname{Ass}(S / I)=\left\{P_{1}, \ldots, P_{s}\right\}$.

According to Lyubeznik [9] the size of $I$, denoted size $(I)$, is the number $a+(n-b)-1$, where $a$ is the minimum number $t$ such that there exist $j_{1}<\cdots<j_{t}$ with

$$
\sqrt{\sum_{l=1}^{t} Q_{j_{l}}}=\sqrt{\sum_{j=1}^{s} Q_{j}},
$$

and where $b=\operatorname{ht}\left(\sum_{j=1}^{s} Q_{j}\right)$. It is clear from the definition that size $(I)$ depends only on the associated prime ideals of $S / I$. In the above definition if we replace "there exists $j_{1}<\cdots<j_{t}$ " by "for all $j_{1}<\cdots<j_{t}$ ", we obtain the definition of bigsize $(I)$, introduced by Popescu [11]. Clearly bigsize $(I) \geq \operatorname{size}(I)$.

Key Words: Monomial ideal, Stanley decomposition, Stanley depth, Lexsegment ideal, Minimal depth.

2010 Mathematics Subject Classification: Primary 13C15, Secondary 13P10, 13F20.

Received: April, 2012.

Revised: May, 2012.

Accepted: February, 2013. 
Theorem 0.1. (Lyubeznik [9]) Let $I \subset S$ ba a monomial ideal then $\operatorname{depth}(I) \geq$ $1+\operatorname{size}(I)$.

Herzog, Popescu and Vladoiu say in [5] that a monomial ideal $I$ has minimal depth, if $\operatorname{depth}(I)=\operatorname{size}(I)+1$. Suppose above that $P_{i} \not \subset \sum_{1=j \neq i}^{s} P_{j}$ for all $i \in[s]$. Then $I$ has minimal depth as shows our Corollary 1.3 which extends [11, Theorem 2.3]. It is easy to see that if $I$ has bigsize 1 then it must have minimal depth (see our Corollary 1.5).

Next we consider the lexicographical order on the monomials of $S$ induced by $x_{1}>x_{2}>\cdots>x_{n}$. Let $d \geq 2$ be an integer and $\mathcal{M}_{d}$ the set of monomials of degree $d$ of $S$. For two monomials $u, v \in \mathcal{M}_{d}$, with $u \geq_{\text {lex }} v$, the set

$$
\mathcal{L}(u, v)=\left\{w \in \mathcal{M}_{d} \mid u \geq_{\text {lex }} w \geq_{\text {lex }} v\right\}
$$

is called a lexsegment set. A lexsegment ideal in $S$ is a monomial ideal of $S$ which is generated by a lexsegment set. We show that a lexsegment ideal has minimal depth (see our Theorem 1.6).

Now, let $M$ be a finitely generated multigraded $S$-module, $z \in M$ be a homogeneous element in $M$ and $z K[Z], Z \subseteq\left\{x_{1}, \ldots, x_{n}\right\}$ the linear $K$ subspace of $M$ of all elements $z f, f \in K[Z]$. Such a linear $K$-subspace $z K[Z]$ is called a Stanley space of dimension $|Z|$ if it is a free $K[Z]$-module, where $|Z|$ denotes the number of indeterminates in $Z$. A presentation of $M$ as a finite direct sum of spaces $\mathcal{D}: M=\bigoplus_{i=1}^{r} z_{i} K\left[Z_{i}\right]$ is called a Stanley decomposition. Stanley depth of a decomposition $\mathcal{D}$ is the number

$$
\operatorname{sdepth} \mathcal{D}=\min \left\{\left|Z_{i}\right|: i=1, \ldots, r\right\} .
$$

The number

$$
\operatorname{sdepth}(M):=\max \{\operatorname{sdepth}(\mathcal{D}): \text { Stanley decomposition of } M\}
$$

is called Stanley depth of $M$. In [14] R. P. Stanley conjectured that

$$
\operatorname{sdepth}(M) \geq \operatorname{depth}(M) .
$$

Theorem $0.2([5])$. Let $I \subset S$ be a monomial ideal then $\operatorname{sdepth}(I) \geq 1+$ size $(I)$. In particular, Stanley's conjecture holds for the monomial ideals of minimal depth.

As a consequence, Stanley's depth conjecture holds for all ideals considered above since they have minimal depth. It is still not known a relation between $\operatorname{sdepth}(I)$ and $\operatorname{sdepth}(S / I)$, but our Theorem 2.3 shows that Stanley's conjecture holds also for $S / I$ if $P_{i} \not \subset \sum_{1=j \neq i}^{s} P_{j}$ for all $i \in[s]$. Some of the recent development about the Stanley's conjecture is given in [12]. 


\section{Minimal depth}

We start this section extending some results of Popescu in [11]. Lemma 1.1, Proposition 1.2, Lemma 1.4 and Corollary 1.5 were proved by Popescu when $I$ is a squarefree monomial ideal. We show that with some small changes the same proofs work even in the non-squarefree case.

Lemma 1.1. Let $I=\bigcap_{i=1}^{s} Q_{i}$ be the irredundant presentation of $I$ as an intersection of primary monomial ideals. Let $P_{i}:=\sqrt{Q_{i}}$. If $P_{s} \not \subset \sum_{i=1}^{s-1} P_{i}$, then

$\operatorname{depth}(S / I)=\min \left\{\operatorname{depth}\left(S / \cap_{i=1}^{s-1} Q_{i}\right), \operatorname{depth}\left(S / Q_{s}\right), 1+\operatorname{depth}\left(S / \cap_{i=1}^{s-1}\left(Q_{i}+Q_{s}\right)\right)\right\}$.

Proof. We have the following exact sequence

$$
0 \longrightarrow S / I \longrightarrow S / \cap_{i=1}^{s-1} Q_{i} \oplus S / Q_{s} \longrightarrow S / \cap_{i=1}^{s-1}\left(Q_{i}+Q_{s}\right) \longrightarrow 0 .
$$

Clearly $\operatorname{depth}(S / I) \leq \operatorname{depth}\left(S / Q_{s}\right)$ by [1, Proposition 1.2.13]. Choosing $x_{j}^{a}$ where $x_{j} \in P_{s} \not \subset \sum_{i=1}^{s-1} P_{i}$ and $a$ is minimum such that $x_{j}^{a} \in Q_{s}$ we see that $I: x_{j}^{a}=\cap_{i=1}^{s-1} Q_{i}$ and by [13, Corollary 1.3$]$ we have

$$
\operatorname{depth}(S / I) \leq \operatorname{depth} S /\left(I: x_{j}^{a}\right)=\operatorname{depth} S /\left(\cap_{i=1}^{s-1} Q_{i}\right) .
$$

Now by using Depth Lemma (see [15, Lemma 1.3.9]) we have

$\operatorname{depth}(S / I)=\min \left\{\operatorname{depth}\left(S / \cap_{i=1}^{s-1} Q_{i}\right), \operatorname{depth}\left(S / Q_{s}\right), 1+\operatorname{depth}\left(S / \cap_{i=1}^{s-1}\left(Q_{i}+Q_{s}\right)\right)\right\}$, which is enough.

Proposition 1.2. Let $I=\bigcap_{i=1}^{s} Q_{i}$ be the irredundant presentation of $I$ as an intersection of primary monomial ideals. Let $P_{i}:=\sqrt{Q_{i}}$. If $P_{i} \not \subset \sum_{1=i \neq j}^{s-1} P_{j}$ for all $i \in[s]$. Then $\operatorname{depth}(S / I)=s-1$.

Proof. It is enough to consider the case when $\sum_{j=1}^{s} P_{j}=\mathfrak{m}$. We use induction on $s$. If $s=1$ the result is trivial. Suppose that $s>1$. By Lemma 1.1 we get

$\operatorname{depth}(S / I)=\min \left\{\operatorname{depth}\left(S / \cap_{i=1}^{s-1} Q_{i}\right), \operatorname{depth}\left(S / Q_{s}\right), 1+\operatorname{depth}\left(S / \cap_{i=1}^{s-1}\left(Q_{i}+Q_{s}\right)\right)\right\}$.

Then by induction hypothesis we have

$$
\operatorname{depth}\left(S / \cap_{i=1}^{s-1} Q_{i}\right)=s-2+\operatorname{dim}\left(S /\left(\sum_{i=1}^{s-1} Q_{i}\right)\right) \geq s-1 .
$$


We see that $\cap_{i=1}^{s-1}\left(Q_{i}+Q_{s}\right)$ satisfies also our assumption, the induction hypothesis gives $\operatorname{depth}\left(S / \cap_{i=1}^{s-1}\left(Q_{i}+Q_{s}\right)\right)=s-2$. Since $Q_{i} \not \subset Q_{s}, i<s$ by our assumption we get $\operatorname{depth}\left(S / Q_{s}\right)>\operatorname{depth}\left(S /\left(Q_{i}+Q_{s}\right)\right)$ for all $i<s$. It follows $\operatorname{depth}\left(S / Q_{s}\right) \geq 1+\operatorname{depth}\left(S / \cap_{i=1}^{s-1}\left(Q_{i}+Q_{s}\right)\right)$ which is enough.

Corollary 1.3. Let $I \subset S$ be a monomial ideal such that $\operatorname{Ass}(S / I)=$ $\left\{P_{1} \ldots, P_{s}\right\}$ where $P_{i} \not \subset \sum_{1=j \neq i}^{s} P_{j}$ for all $i \in[s]$. Then I has minimal depth.

Proof. Clearly $\operatorname{size}(I)=s-1$ and by Proposition 1.2 we have $\operatorname{depth}(I)=s$, thus we have $\operatorname{depth}(I)=\operatorname{size}(I)+1$, i.e. $I$ has minimal depth.

Lemma 1.4. Let $I=\cap_{i=1}^{s} Q_{i}$ be the irredundant primary decomposition of $I$ and $\sqrt{Q_{i}} \neq \mathfrak{m}$ for all $i$. Suppose that there exists $1 \leq r<s$ such that $\sqrt{Q_{i}+Q_{j}}=\mathfrak{m}$ for each $r<j \leq s$ and $1 \leq i \leq r$. Then $\operatorname{depth}(I)=2$.

Proof. The proof follows by using Depth Lemma on the following exact sequence.

$$
0 \longrightarrow S / I \longrightarrow S / \cap_{i=1}^{r} Q_{i} \oplus S / \cap_{j>r}^{s} Q_{j} \longrightarrow S / \cap_{i=1}^{r} \cap_{j>r}^{s}\left(Q_{i}+Q_{j}\right) \longrightarrow 0 .
$$

Corollary 1.5. Let $I \subset S$ be a monomial ideal. If bigsize of $I$ is one then $I$ has minimal depth.

Proof. We know that $\operatorname{size}(I) \leq \operatorname{bigsize}(I)$. If $\operatorname{size}(I)=0$ the $\operatorname{depth}(I)=1$ and the result follows in this case. Now let us suppose that $\operatorname{size}(I)=1$. By Lemma 1.4 we have $\operatorname{depth}(I)=2$. Hence the result follows.

Let $d \geq 2$ be an integer and $\mathcal{M}_{d}$ the set of monomials of degree $d$ of $S$. For two monomials $u, v \in \mathcal{M}_{d}$, with $u \geq_{\text {lex }} v$, we consider the lexsegment set

$$
\mathcal{L}(u, v)=\left\{w \in \mathcal{M}_{d} \mid u \geq_{\text {lex }} w \geq_{\text {lex }} v\right\} .
$$

Theorem 1.6. Let $I=(\mathcal{L}(u, v)) \subset S$ be a lexsegment ideal. Then $\operatorname{depth}(I)=$ $\operatorname{size}(I)+1$, that is $I$ has minimal depth.

Proof. For the trivial cases $u=v$ the result is obvious. Suppose that $u=$ $x_{1}^{a_{1}} \cdots x_{n}^{a_{n}}$

$v=x_{1}^{b_{1}} \cdots x_{n}^{b_{n}} \in S$. First assume that $b_{1}=0$. If there exist $r$ such that $a_{1}=\cdots=a_{r}=0$ and $a_{r+1} \neq 0$, then $I$ is a lexsegment ideal in $S^{\prime}:=$ $K\left[x_{r+1}, \ldots, x_{n}\right]$. We get $\operatorname{depth}(I S)=\operatorname{depth}\left(I S^{\prime}\right)+r$ and by definition of size we have size $(I S)=\operatorname{size}\left(I S^{\prime}\right)+r$. This means that without loss of generality we can assume that $a_{1}>0$. If $x_{n} u / x_{1} \geq_{l e x} v$, then by [3, Proposition 3.2] $\operatorname{depth}(I)=1$ which implies that $\mathfrak{m} \in \operatorname{Ass}(S / I)$, thus size $(I)=0$ and the result 
follows in this case. Now consider the complementary case $x_{n} u / x_{1}<_{\text {lex }} v$, then $u$ is of the form $u=x_{1} x_{l}^{a_{l}} \cdots x_{n}^{a_{n}}$ where $l \geq 2$. Let $I=\cap_{i=1}^{s} Q_{i}$ be an irredundant primary decomposition of $I$, where $Q_{i}^{\prime} s$ are monomial primary ideals. If $l \geq 4$ and $v=x_{2}^{d}$ then by [3, Proposition 3.4] we have $\operatorname{depth}(I)=l-1$. After [6, Proposition 2.5(ii)] we know that

$$
\sqrt{\sum_{i=1}^{s} Q_{i}}=\left(x_{1}, x_{2}, x_{l}, \ldots, x_{n}\right) \notin \operatorname{Ass}(S / I),
$$

but $\left(x_{1}, x_{2}\right),\left(x_{2}, x_{l}, \ldots, x_{n}\right) \in \operatorname{Ass}(S / I)$. Therefore, $\operatorname{size}(I)=l-2$ and we have $\operatorname{depth}(I)=\operatorname{size}(I)+1$, so we are done in this case. Now consider the case $v=x_{2}^{d-1} x_{j}$ for some $3 \leq j \leq n-2$ and $l \geq j+2$, then again by [3, Proposition 3.4] we have $\operatorname{depth}(I)=l-j+1$ and by [6, Proposition 2.5(ii)] we have

$$
\sqrt{\sum_{i=1}^{s} Q_{i}}=\left(x_{1}, \ldots, x_{j}, x_{l}, \ldots, x_{n}\right) \notin \operatorname{Ass}(S / I)
$$

and $\left(x_{1}, \ldots, x_{j}\right),\left(x_{2}, \ldots, x_{j}, x_{l}, \ldots, x_{n}\right) \in \operatorname{Ass}(S / I)$. Therefore, $\operatorname{size}(I)=l-j$ and again we have $\operatorname{depth}(I)=\operatorname{size}(I)+1$. Now for all the remaining cases by $[3$, Proposition 3.4] we have $\operatorname{depth}(I)=2$, and by [6, Proposition 2.5(i)]

$$
\sqrt{\sum_{i=1}^{s} Q_{i}}=\left(x_{1}, \ldots, x_{n}\right) \notin \operatorname{Ass}(S / I),
$$

but $\left(x_{1}, \ldots, x_{j}\right),\left(x_{2}, \ldots, x_{n}\right) \in \operatorname{Ass}(S / I)$, for some $j \geq 2$. Therefore size $(I)=$ 1. Thus the equality $\operatorname{depth}(I)=\operatorname{size}(I)+1$ follows in all cases when $b_{1}=0$.

Now let us consider that $b_{1}>0$, then $I=x_{1}^{b_{1}} I^{\prime}$ where $I^{\prime}=\left(I: x_{1}^{b_{1}}\right)$. Clearly $I^{\prime}$ is a lexsegment ideal generated by the lexsegment set $\mathcal{L}\left(u^{\prime}, v^{\prime}\right)$ where $u^{\prime}=u / x_{1}^{b_{1}}$ and $v^{\prime}=v / x_{1}^{b_{1}}$. The ideals $I, I^{\prime}$ are isomorphic, therefore $\operatorname{depth}\left(I^{\prime}\right)=\operatorname{depth}(I)$. It is enough to show that $\operatorname{size}\left(I^{\prime}\right)=\operatorname{size}(I)$. We have the exact sequence

$$
0 \rightarrow S / I^{\prime} \stackrel{x_{1}^{b_{1}}}{\rightarrow} S / I \rightarrow S /\left(I, x_{1}^{b_{1}}\right)=S /\left(x_{1}^{b_{1}}\right) \rightarrow 0,
$$

and therefore

$$
\operatorname{Ass}\left(S / I^{\prime}\right) \subset \operatorname{Ass}(S / I) \subset \operatorname{Ass}\left(S / I^{\prime}\right) \cup\left\{\left(x_{1}\right)\right\} .
$$

As $\left\{\left(x_{1}\right)\right\} \in \operatorname{Ass}(S / I)$ since it is a minimal prime over $I$, we get $\operatorname{Ass}(S / I)=$ $\operatorname{Ass}\left(S / I^{\prime}\right) \cup\left\{\left(x_{1}\right)\right\}$. Let $s^{\prime}$ be the minimum number such that there exist $P_{1}, \ldots, P_{s} \in \operatorname{Ass} S / I^{\prime}$ such that $\sum_{i=1}^{s} P_{i}=a:=\sum_{P \in \operatorname{Ass}\left(S / I^{\prime}\right)} P$. Then 
$\operatorname{size}\left(I^{\prime}\right)=s^{\prime}+\operatorname{dim}(S / a)-1$. Let $s$ be the minimum number $t$ such that there exist $t$ prime ideals in $\operatorname{Ass}(S / I)$ whose sum is $\left(a, x_{1}\right)$. By [6, Lemma 2.1] we have that atleast one prime ideal from $\operatorname{Ass}\left(S / I^{\prime}\right)$ contains necessarily $x_{1}$, we have $x_{1} \in a$. It follows $s \leq s^{\prime}$ because anyway $\sum_{i=1}^{s^{\prime}} P_{i}=a=\sum_{P \in \operatorname{Ass}(S / I)} P$. If we have $P_{1}^{\prime}, \ldots, P_{s-1}^{\prime} \in \operatorname{Ass}\left(S / I^{\prime}\right)$ such that $\sum_{i=1}^{s-1} P_{i}^{\prime}+\left(x_{1}\right)=a$ then we have also $\sum_{i=1}^{s-1} P_{i}^{\prime}+P_{1}=a$ for some $P_{1} \in \operatorname{Ass}\left(S / I^{\prime}\right)$ which contains $x_{1}$. Thus $s=s^{\prime}$ and so $\operatorname{size}(I)=\operatorname{size}\left(I^{\prime}\right)$.

\section{Stanley depth of cyclic modules defined by ideals of minimal depth}

Using Corollaries 1.3, 1.5 and Theorems 1.6, 0.2 we get the following theorem.

Theorem 2.1. Stanley's conjecture holds for I, if it satisfies one of the following statements:

1. $P_{i} \nsubseteq \sum_{1=j \neq i}^{s} P_{j}$ for all $i \in[s]$,

2. the bigsize of $I$ is one,

3. I is a lexsegment ideal.

Remark 2.2. Usually, if Stanley's conjecture holds for an ideal $I$ then we may show that it holds for the module $S / I$ too. There exist no general explanation for this fact. If $I$ is a monomial ideal of bigsize one then Stanley's conjecture holds for $S / I$. Indeed, case $\operatorname{depth}(S / I)=0$ is trivial. Suppose $\operatorname{depth}(S / I) \neq$ 0 , then by Lemma $1.4 \operatorname{depth}(S / I)=1$, therefore by [2, Proposition 2.13] $\operatorname{sdepth}(S / I) \geq 1$. If $I$ is a lexsegment ideal then Stanley's conjecture holds for $S / I[6]$. Below we show this fact in the first case of the above theorem.

Theorem 2.3. Let $I=\bigcap_{i=1}^{s} Q_{i}$ be the irredundant presentation of $I$ as an intersection of primary monomial ideals. Let $P_{i}:=\sqrt{Q_{i}}$. If $P_{i} \not \subset \sum_{1=i \neq j}^{s} P_{j}$ for all $i \in[s]$ then $\operatorname{sdepth}(S / I) \geq \operatorname{depth}(S / I)$, that is the Stanley's conjecture holds for $S / I$.

Proof. Using [4, Lemma 3.6] it is enough to consider the case $\sum_{i=1}^{s} P_{i}=\mathfrak{m}$. By Proposition 1.2 we have $\operatorname{depth}(S / I)=s-1$. We show that $\operatorname{sdepth}(S / I)$ $\geq s-1$. Apply induction on $s$, case $s=1$ being clear. Fix $s>1$ and apply induction on $n$. If $n \leq 5$ then the result follows by [10]. Let $A:=\cup_{i=1}^{s}\left(G\left(P_{i}\right) \backslash\right.$ $\left.\sum_{1=j \neq i}^{s} G\left(P_{j}\right)\right)$. If $(\bar{A})=\mathfrak{m}$ then note that $G\left(P_{i}\right) \cap G\left(P_{j}\right)=\emptyset$ for all $i \neq j$. By [7, Theorem 2.1] and [8, Theorem 3.1] we have $\operatorname{sdepth}(S / I) \geq s-1$. Now suppose that $(A) \neq \mathfrak{m}$. By renumbering the primes and variables we can 
assume that $x_{n} \notin A$. There exists a number $r, 2 \leq r \leq s$ such that $x_{n} \in G\left(P_{j}\right)$, $1 \leq j \leq r$ and $x_{n} \notin G\left(P_{j}\right), r+1 \leq j \leq s$. Let $S^{\prime}:=K\left[x_{1}, \ldots, x_{n-1}\right]$. First assume that $r<s$. Let $Q_{j}^{\prime}=Q_{j} \cap S^{\prime}, P_{j}^{\prime}=P_{j} \cap S^{\prime}$ and $J=\bigcap_{i=r+1}^{s} Q_{i}^{\prime} \subset S^{\prime}$, $L=\bigcap_{i=1}^{r} Q_{i}^{\prime} \subset S^{\prime}$. We have $\left(I, x_{n}\right)=\left((J \cap L), x_{n}\right)$ because $\left(Q_{j}, x_{n}\right)=$ $\left(Q_{j}^{\prime}, x_{n}\right)$ using the structure of monomial primary ideals given in [15]. In the exact sequence

$$
0 \longrightarrow S /\left(I: x_{n}\right) \longrightarrow S / I \longrightarrow S /\left(I, x_{n}\right) \longrightarrow 0,
$$

the sdepth of the right end is $\geq s-1$ by induction hypothesis on $n$ for $J \cap L \subset S^{\prime}$ (note that we have $P_{i}^{\prime} \not \subset \sum_{1=i \neq j}^{s} P_{j}^{\prime}$ for all $i \in[s]$ since $x_{n} \notin A$ ). Let $e_{I}$ be the maximum degree in $x_{n}$ of a monomial from $G(I)$. Apply induction on $e_{I}$. If $e_{I}=1$ then $\left(I: x_{n}\right)=J S$ and the sdepth of the left end in the above exact sequence is equal with $\operatorname{sdepth}(S / J S) \geq(s-r-1)+r=s-1$ since there are at least $r$ variables which do not divide the minimal monomial generators of ideal $\left(I: x_{n}\right)$ and we may apply induction hypothesis on $s$ for $J$. By [13, Theorem 3.1] we have $\operatorname{sdepth}(S / I) \geq \min \left\{\operatorname{sdepth}\left(S /\left(I: x_{n}\right)\right), \operatorname{sdepth}\left(S /\left(I, x_{n}\right)\right)\right\} \geq$ $s-1$. If $e_{I}>1$ then note that $e_{\left(I: x_{n}\right)}<e_{I}$ and by induction hypothesis on $e_{I}$ or $s$ we get $\operatorname{sdepth}\left(S /\left(I: x_{n}\right)\right) \geq s-1$. As above we obtain by [13, Theorem 3.1] $\operatorname{sdepth}(S / I) \geq s-1$.

Now let $r=s$. If $e_{I}=1$ then $I=\left(L, x_{n}\right)$ and by induction on $n$ we have $\operatorname{sdepth}(S / I)=\operatorname{sdepth}\left(S^{\prime} / L\right) \geq s-1$. If $e_{I}>1$ then by induction hypothesis on $e_{I}$ and $s$ we get $\operatorname{sdepth}\left(S /\left(I: x_{n}\right)\right) \geq s-1$. As above we are done using [13, Theorem 3.1].

\section{Acknowledgement}

This research is partially supported by National University of Sciences and Technology (NUST), Islamabad, and a grant of Higher Education Commission of Pakistan Ref. No: PM-IPFP/HRD/HEC/2012/2778.

\section{References}

[1] W. Bruns and J. Herzog, Cohen-Macaulay rings. Rev. ed. Cambridge Studies in Advanced Mathematics 39, Cambridge University Press 1998.

[2] W. Bruns, C. Krattenthaler, J. Uliczka, Stanley decompositions and Hilbert depth in the Koszul complex, J. Commut. Algebra 2(2010), 327357.

[3] V. Ene, A.Olteanu, L. Sorrenti, Properties of lexsegment ideals, Osaka J. Math., 47, 2010, 1-21. 
[4] J. Herzog, M. Vladoiu, X. Zheng, How to compute the Stanley depth of a monomial ideal, J. Algebra, 322(9)(2009), 3151-3169.

[5] J. Herzog, D. Popescu, M. Vlădoiu, Stanley depth and size of a monomial ideal, to appear in Proceed. AMS.

[6] M. Ishaq, Lexsegment ideals are sequentially Cohen-Macaulay, to appear in Algebra Colloquium. arXiv:1010.5615v3.

[7] M. Ishaq, Values and bounds for the Stanley depth, Carpathian J. Math., 27 (2011), No.2, 217-224.

[8] M. Ishaq, M.I. Qureshi, Upper and lower bounds for the Stanley depth of certain classes of monomial ideals and their residue class rings, Communications in Algebra, 41(2013), no.3, 1107-1116.

[9] G. Lyubeznik, On the Arithmetical Rank of Monomial ideals. J. Algebra 112, 8689 (1988).

[10] D. Popescu, Stanley depth of multigraded modules, J. Algebra 321(2009), 2782-2797.

[11] D. Popescu, Stanley conjecture on intersection of four monomial prime ideals, to appear in Communications in Algebra, arXiv:1009.5646v1.

[12] D. Popescu, Bounds of Stanley depth, Analele Univ. Ovidius Constanta, 19(2)(2011), 187-194.

[13] A. Rauf, Depth and Stanley depth of multigraded modules, Comm. Algebra, 38 (2010), 773-784.

[14] R.P. Stanley, Linear Diophantine equations and local cohomology, Invent. Math. 68 (1982). 175-193.

[15] R. H. Villarreal, Monomial Algebras, New York: Marcel Dekker Inc. 2001.

Muhammad ISHAQ,

Department of Mathematics,

School of Natural Sciences,

National University of Sciences and Technology Islamabad,

Sector, H-12, 44000 Islamabad, Pakistan.

Email: ishaq_maths@yahoo.com 